\title{
EVOLUTION OF THE BRAIN COMPUTING INTERFACE (BCI) AND PROPOSED ELECTROENCEPHALOGRAPHY (EEG) SIGNALS BASED AUTHENTICATION MODEL
}

\author{
Qaseem Ramzan ${ }^{1,2 *}$, Stanislav Shidlovskiy ${ }^{1}$ \\ ${ }^{1}$ National Research Tomsk State University. 634050, Tomsk, Russia \\ ${ }^{2}$ toggleTechs (a creativity platform), Islamabad, Pakistan
}

\begin{abstract}
With current advancements in the field of Brain Computer interface it is required to study how it will affect the other technologies currently in use. In this paper, the authors motivate the need of Brain Computing Interface in the era of IoT (Internet of Things), and analyze how $\mathrm{BCI}$ in the presence of IoT could have serious privacy breach if not protected by new kind of more secure protocols. Security breach and hacking has been around for a long time but now we are sensitive towards data as our lives depend on it. When everything is interconnected through IoT and considering that we control all interconnected things by means of our brain using BCI (Brain Computer Interface), the meaning of security breach becomes much more sensitive than in the past. This paper describes the old security methods being used for authentication and how they can be compromised. Considering the sensitivity of data in the era of IoT, a new form of authentication is required, which should incorporate BCI rather than usual authentication techniques.
\end{abstract}

\section{What is a Brain Computer Interface?}

What is a Brain Computer Interface (BCI)? A brain computer interface, sometimes called a mind-machine interface (MMI), direct neural interface (DNI), or brain-machine interface (BMI), is a direct communication pathway between an enhanced or wired brain and an external device. BCIs are often directed at researching, mapping, assisting, augmenting, or repairing human cognitive or sensory-motor functions [1].

\subsection{History of BCI Research}

In the 1970s, research on BCIs started at the University of California, which led to the emergence of the expression brain-computer interface. The focus of BCI research and development continues to be primarily on neuroprosthetics applications that can help to restore

\footnotetext{
* Corresponding author: qaseemramzan0@gmail.com
} 
damaged sight, hearing, and movement. The mid-1990s marked the appearance of the first neuroprosthetic devices for humans. BCI does not read the mind accurately, but detects the smallest of changes in the energy radiated by the brain when you think in a certain way. A BCI recognizes specific energy/ frequency patterns in the brain.

John Donoghue and his team of researchers from Brown University formed a public traded company, Cyberkinetics, in 2001. The goal was to design a brain computer interface, the socalled BrainGate, commercially. The company has come up with NeuroPort TM, which was its first commercial product. Researchers from Columbia University Medical Center have successfully monitored and recorded electrical activity in the brain with improved precision. According to researchers, NeuroPort ${ }^{\mathrm{TM}}$ Neural Monitoring System enabled them to identify micro-seizure activity prior to epileptic seizures among patients [2].

\subsection{Current Standing of BCl}

BCIs comprise an active area of research and could start to integrate advances from adjacent fields such as neuroscience, nanomaterials, electronics miniaturization, and machine learning. For example, one neuro-imaging research project is starting to make guesses as to what participants see during brain scans, purporting to be able to distinguish between a cat and a person. Merging this kind of functionality with BCIs might produce new applications. Other experimental BCI projects have been proposed. One is Neocortical Brain-Cloud Interface: autonomous nanorobots that could connect to axons and neuronal synaptic clefts, or embed themselves into the peripheral calvaria and pericranium of the skull. Another project, Brainets, envisions linking multiple organic computing units (brains) to silicon computing networks. The third project is Neural Dust, in which thousands of 10-100 micron-sized free-floating sensor nodes would reside in the brain and provide a computing processing network [3].

\subsection{Future of $\mathrm{BCl}$}

A number of developments have been taking place in the field. By 2050, it is has been suggested that BCI could become a magic wand, helping men to control objects with their mind. The day isn't far off when man may be able to guide an outside object with their thoughts in order to consistently execute both natural and complex motions of everyday life.

So far BCIs have been conceived primarily as a solution for medical pathologies. However, it is possible to see BCIs more expansively as a platform for cognitive enhancement and human-machine collaboration. The BCI functionality of typing on a keyboard with your mind suggests the possibility of having an always-on brain-Internet connection. Consider what the world might be like if each individual had a live $24 / 7$ brain connection to the Internet. Just as cell phones connected individual people to communications networks, BCIs might similarly connect individual brains to communications networks. We propose a variety of $\mathrm{BCI}$ applications and concepts throughout the rest of this paper, all of which are speculative.

In one sense, ubiquitous BCIs are expected. It is contemplated that communication technology, already mobilized to the body via the cell phone, could be "brought on board" even more pervasively. BCIs are merely a next-generation improvement to the current situation of people constantly staring at their phones. In another sense, though, BCIs are not only a "better horse" technology: they are also a "car" in that it is impossible to foresee the full range of future applications that might be enabled from the present moment. BCIs pose a variety of practical, ethical, and philosophical issues. Life itself and the definition of what it is to be human could be quite different in a world where BCIs are widespread. Some of the immediate practical concerns of BCIs could include invasiveness, utility, reversibility, support, maintenance, upgradability (hardware and software), anti-hacking and anti-virus 
protection, cost, and accessibility. Beyond practical concerns, there are ethical issues regarding privacy and security. For example, neural data privacy rights are an area where standards need to be defined. There could be at least three classes of BCI applications introduced in graduated phases of risk and complexity: biological cure and enhancement; information and entertainment; and self-actualization (realization of individual cognitive and artistic potential). Each of these is for separate discussion [4].

\section{4 $\mathrm{BCl}$ in view of loT (Internet of Things)}

The IoT (Internet of Things) is a network of physical devices, vehicles, home appliances, and other items embedded with electronics, software, sensors, actuators, and network connectivity, which enable these objects to connect and exchange data. Each thing is uniquely identifiable through its embedded computing system but is able to inter-operate within the existing Internet infrastructure. Experts estimate that the IoT will consist of about 30 billion objects by $2020[5]$.

The advancement of consumer electroencephalography (EEG) devices opens the technology up to a completely new world of BCI options. Combining EEG technologies with other IoT technologies like heart rate monitoring, facial emotion recognition, smart homes, home security systems, and personal devices will put new threats of privacy and security breach.

\subsection{Operation principles of $\mathrm{BCI}$}

The purpose of a BCI is to detect and quantify features of brain signals that indicate the user's intentions and to translate these features in real time into device commands that accomplish the user's intent. To achieve this, a BCI system consists of four sequential components.

1. Signal Acquisition.

2. Feature Extraction.

3. Feature Translation.

4. Device Output.

These four components are controlled by an operating protocol that defines the onset and timing of operation, the details of signal processing, the nature of the device commands, and the oversight of performance. An effective operating protocol allows a BCI system to be flexible and to serve the specific needs of each user.

Electrical signals from brain activity are detected by recording electrodes located on the scalp, on the cortical surface, or within the brain. The brain signals are amplified and digitized. Pertinent signal characteristics are extracted and then translated into commands that control an output device, such as a spelling program, a motorized wheelchair, or a prosthetic limb. Feedback from the device enables the user to modify the brain signals in order to maintain effective device performance. BCI is brain-computer interface; ECoG is electrocorticography; EEG is electroencephalography [6].

\subsubsection{Signal Acquisition}

Signal acquisition is the measurement of brain signals using a particular sensor modality (e.g., scalp or intracranial electrodes for electrophysiologic activity, fMRI for metabolic activity). The signals are amplified to levels suitable for electronic processing (and they may also be subjected to filtering to remove electrical noise or other undesirable signal characteristics, such as $60-\mathrm{Hz}$ power line interference). The signals are then digitized and transmitted to a computer. 


\subsubsection{Feature Extraction}

Feature extraction is the process of analyzing the digital signals to distinguish pertinent signal characteristics (i.e. signal features related to a person's intent) from extraneous content and representing them in a compact form suitable for translation into output commands. These features should have strong correlations with the user's intent. Because much of the relevant (i.e. most strongly correlated) brain activity is either transient or oscillatory, the most commonly extracted signal features in current BCI systems are time-triggered EEG or ECoG (Electrocorticography) response amplitudes and latencies, power within specific EEG or ECoG frequency bands, or firing rates of individual cortical neurons. Environmental artifacts and physiologic artifacts such as EMG (Electromyographic) signals are avoided or removed to ensure accurate measurement of the brain signal features.

\subsubsection{Device Output}

The commands from the feature translation algorithm operate the external device, providing functions such as letter selection, cursor control, robotic arm operation, and so forth. The device operation provides feedback to the user, thus closing the control loop.

\subsubsection{Feature Translation}

The resulting signal features are then passed to the feature translation algorithm, which converts the features into the appropriate commands for the output device (i.e. commands that accomplish a user's intent). For example, a power decrease in a given frequency band could be translated into an upward displacement of a computer cursor, or a P300 potential could be translated into selection of the letter that evoked it. The translation algorithm should be dynamic to accommodate and adapt to spontaneous or learned changes in the signal features and to ensure that the user's possible range of feature values covers the full range of device control.

\subsection{Programming Using OpenBCl}

OpenBCI is an open source brain-computer interface platform created by Joel Murphy and Conor Russomanno after a successful Kickstarter campaign in late 2013. OpenBCI boards can be used to measure and record electrical activity produced by the brain (EEG), muscles (EMG), and heart (EKG), and is compatible with standard EEG electrodes. The OpenBCI boards can be used with the open source OpenBCI GUI, or they can be integrated with other open-source EEG signal processing tools.

\subsubsection{Hardware}

The OpenBCI 32-bit Board uses the ADS1299, an IC developed by Texas Instruments for biopotential measurements. The OpenBCI uses a microcontroller for on-board processing the 8-bit version (now deprecated) uses an Arduino-compatible ATmega328P IC, while the 32-bit board uses a PIC microcontroller - and can write the EEG data to an SD card, or transmit it to software on a computer over a bluetooth link. In 2015, OpenBCI announced the Ganglion board with a 2 nd Kickstarter campaign. It costs $\$ 100$ (1/5 the cost the 32-bit board). It has four input channels for measuring EEG, EMG, and EKG, and is also bluetooth enabled [7]. Table 1 presents price details of OpenBCI Hardware. 
Table 1. Price Details of OpenBCI Hardware (as of December 2017)

\begin{tabular}{|c|c|c|}
\hline Index & Hardware & Price (\$) \\
\hline 1 & Gangolion Board (4-channels) & 199.99 \\
\hline 2 & $\begin{array}{c}\text { Cyton Biosensing Board (8- } \\
\text { channels) }\end{array}$ & 499.99 \\
\hline 3 & $\begin{array}{c}\text { Cyton + Daisy Biosensing Board } \\
\text { (16-channels) }\end{array}$ & 949.99 \\
\hline 4 & WiFi Sheild & 149.99 \\
\hline 5 & Ultra Cortex Mark "IV" EEG \\
Headset & 349.99 \\
\hline 6 & Gold Cup Electrodes & 29.99 \\
\hline
\end{tabular}

\subsubsection{Software}

OpenBCI has released an open-source application for use with the OpenBCI written with Processing. Display and processing software written in NodeJS and Python are also being developed [8]. Table 2 presents the major open-source repositories of OpenBCI.

Table 2. Major Open-Source Repositories of OpenBCI (as of December 2017)

\begin{tabular}{|c|c|c|}
\hline Index & Repositories & Details \\
\hline 1 & OpenBCI_GUI & $\begin{array}{c}\text { OpenBCI_GUI is a cross platform } \\
\text { application for the OpenBCI Cyton and } \\
\text { Ganglion. Tested on Mac, Windows and } \\
\text { Ubuntu/Mint Linux. Ganglion requires } \\
\text { some extra configuration on } \\
\text { Linux/Windows. }\end{array}$ \\
\hline 2 & OpenBCI_WIFI & $\begin{array}{c}\text { Firmware library that runs on the Push } \\
\text { The World WiFi Shield to stream data } \\
\text { from OpenBCI Boards }\end{array}$ \\
\hline 3 & OpenBCI_HUB & An electron application for the ganglion. \\
\hline 4 & OpenBCI_WiFi_Sheild & Schematics for the OpenBCI wifi shield \\
\hline 5 & OpenBCI_NodeJS_WiFi & NodeJS driver for the Wifi Shield \\
\hline 6 & OpenBCI_NodeJS & $\begin{array}{c}\text { Node.js SDK for the OpenBCI Biosensor } \\
\text { Board written by Push The World }\end{array}$ \\
\hline 7 & OpenBCI_32bit_Library & $\begin{array}{c}\text { Repository for OpenBCI 32bit Arduino } \\
\text { Libraries }\end{array}$ \\
\hline 8 & OpenBCI_JavaScript_Utilities & Utilities for OpenBCI boards \\
\hline 9 & OpenBCI_NodeJS_Cyton & $\begin{array}{c}\text { The official NodeJS driver for the Cyton } \\
\text { board over Serial. }\end{array}$ \\
\hline
\end{tabular}

\section{Security Challenges in The Era of loT}

This paper proposes a need and plan of switching to more secure authentication methods for the age of Brain Computer Interface and IoT. This new security method should use BCI as a source of authentication method instead of old authentication methods. In the proposed method, authentication should be checked by the EEG Signals from brain and authentication should be granted by verifying the pattern with previously stored pattern. 


\subsection{Authentication Methods Being Used Currently}

Authentication is an essential element of a typical security model. It is a process aimed to confirm identification of a user (in some cases, a machine) that is trying to access resources or $\log$ in. There is a number of different authentication mechanisms, but all serve this same purpose. Some of the security methods being used for Secure Authentication these days are listed below.

1. Passwords.

2. PIN Code.

3. Finger Print.

4. Pattern Recognition Systems.

5. 2-factor Authentication.

Security of all these methods could be compromised one way or the other. There is a proven history of the breach of security with all specified methods.

\section{A Look into BCl Authentication Methods}

BCI Authentication methods will use the pure EEG or EEG + EMG signals to authenticate a user over computer or mobile devices. This will allow the user to be authenticated by brain signals safely without using any other input methods. Such authentication system will be based on EEG signals.

\subsection{Electroencephalogram (EEG) Model for Authentication}

Electroencephalography (EEG) is the recording of electrical activity along the scalp through measuring voltage fluctuations accompanying neurotransmission activity within the brain. The electrodes are attached in a cap-like device. It has unique usability advantages over other types of brain signal recording that recommend it for commercial use. It is portable, inexpensive, and easy to use. EEG recording also provides high temporal resolution. However, its signal to noise ratio and spatial resolution represent a limitation compared to other methods [9].

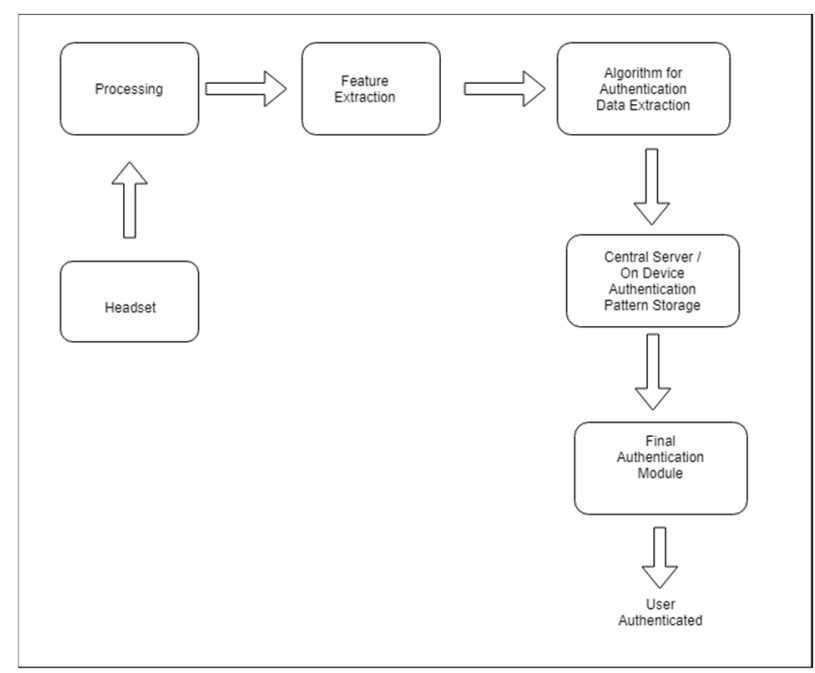

Fig. 1. Proposed Authentication Model Using EEG Signals. 
Figure 1 shows an authentication model using EEG signals proposed by the authors. Signals acquired from headset will be processed and then the important features will be extracted from the raw EEG signals. A custom authentication algorithm extracts important authentication related information and makes a package, which could be checked for authentication. This authentication data package is then compared with previously stored data pattern on either a server or local storage in order to verify if that it is the same user leading to authentication or rejection of user.

\subsection{Current Challenges}

Currently there is a number of challenges causing difficulty for the research process. Once the research is successful, still there will be some limitations to produce this as a locally useable device.

\subsubsection{Usability Challenge}

The headsets we can find currently in the market are costly due to expensive sensors. These devices cannot be used directly and require to setup the electrodes and put a conducting gel on the scalp of a person for signals reading.

\subsubsection{Training Process \& ITR (Information Transfer Rate)}

Training processes express the limitations facing the user acceptance of BCI technology. They include the issues related to the training process necessary for class discrimination. ITR (Information transfer rate) is one of the system evaluation metrics that combine both performance and acceptance aspects [10-13].

Users training is a time-consuming activity either in guiding the user through the process or in the number of recorded sessions. It takes place either in preliminary phase or in the classifier calibration phase. The user is taught to deal with the system as well as to control his or her brain feedback signals in the preliminary phase, while in the calibration phase the trained subject's signal has been used to learn the used classifier.

ITR (Information Transfer Rate) is a widely used evaluation metric for command BCI systems. It depends on the number of choices, the accuracy of target detection, and the average time for a selection. Thus compared to imagery BCI, selective attention strategies achieve higher ITR as their offered choices are larger.

\subsubsection{Non-linearity}

The brain is a highly complex nonlinear system, in which chaotic behavior of neural ensembles can be detected. Thus, EEG signals can be better characterized by nonlinear dynamic methods than linear methods. The training sets are relatively small because usability issues influence the training process. Although heavily training sessions are considered time consuming and demanding for the subjects, they provide the user with necessary experience to deal with the system and learn to control his/her neurophysiological signals. Thus, a significant challenge in designing a BCI is to balance the trade-off between the technological complexity of interpreting the user's brain signals and the amount of training needed for successful operation of the interface [14]. 


\section{Conclusion}

Brain Computer Interface is a newly emerging technology, which is soon going to surround all other technologies and will be involved in daily life activities. It can be easily understood that BCI is going to integrate with IoT on very large scale and this integration will be a new rise in the technological advancements. At the same time, there is a need to find new authentication methods, which are more secure and do not use conventional authentication techniques but rely totally on EEG \& EMG Signals of Brain Computing Interface.

\section{Acknowledgements}

The research presented in this paper was supported by the Russian Foundation for Basic Research (grant No 16-29-04388/17). The authors are grateful to Tatiana B. Rumyantseva from Tomsk State University for English language editing.

\section{References}

[1] Brain Computer Interface. URL: https://en.wikipedia.org/wiki/Brain-computer_interface
[2] History of Brain Computer Interfaces.
URL:

http://www.brainvision.co.uk/blog/2014/04/the-brief-history-of-brain-computerinterfaces

[3] Future of Brain Computing Interfaces (Block-chaining Your Way into a Cloud Mind). URL: http://jetpress.org/v26.2/swan.htm

[4] Melanie Swan. Future of Brain Computing Interfaces (Block-chaining Your Way into a Cloud Mind). URL: http://jetpress.org/v26.2/swan.htm

[5] Internet of Things.URL: https://en.wikipedia.org/wiki/Internet_of_things

[6] Jerry J. Shih. Brain-Computer Interfaces in Medicine [Electronic resource] URL: https://www.ncbi.nlm.nih.gov/pmc/articles/PMC3497935/

[7] OpenBCI. URL: https://en.wikipedia.org/wiki/OpenBCI

[8] OpenBCI Repositories. URL: https://github.com/OpenBCI

[9] Sarah N. Abdulkader. Egyptian Informatics Journal. Brain Computer Interface Applications and Challenges. URL: http://www.sciencedirect.com/science/article/pii/S1110866515000237

[10] Sarah N. Abdulkader. Egyptian Informatics Journal. Information Transfer Rate. URL: http://www.sciencedirect.com/science/article/pii/S1110866515000237

[11] V. Syryamkin, V. Shidlovsky. Correlation-extremal direction-finding systems (Tomsk State University publishing house, 2010)

[12] D.V. Shashev, S.V. Shidlovskiy, Opt. Inst. D. Proc. 51, 227 (2015)

[13] D.V. Shashev, MATEC Web Conf. 79, (2016)

[14] Sarah N. Abdulkader. Egyptian Informatics Journal. Non-Linearity. URL: http://www.sciencedirect.com/science/article/pii/S1110866515000237 